\title{
One half of almost symmetric numerical semigroups
}

\author{
F. Strazzanti * \\ Dipartimento di Matematica, Università degli Studi di Pisa, \\ Largo Bruno Pontecorvo 5, 56127 Pisa, Italy
}

\begin{abstract}
Let $S, T$ be two numerical semigroups. We study when $S$ is one half of $T$, with $T$ almost symmetric. If we assume that the type of $T, t(T)$, is odd, then for any $S$ there exist infinitely many such $T$ and we prove that $1 \leq t(T) \leq 2 t(S)+1$. On the other hand, if $t(T)$ is even, there exists such $T$ if and only if $S$ is almost symmetric and different from $\mathbb{N}$; in this case the type of $S$ is the number of even pseudo-Frobenius numbers of $T$. Moreover, we construct these families of semigroups using the numerical duplication with respect to a relative ideal.
\end{abstract}

MSC: 20M14; 13H10.

Keywords Numerical semigroup - One half of a semigroup · Almost symmetric semigroup · Numerical duplication · type.

\section{Introduction}

A numerical semigroup $S$ is a submonoid of $\mathbb{N}$ such that $\mathbb{N} \backslash S$ is finite. Numerical semigroups arise in several contexts, such as commutative algebra, algebraic geometry, coding theory, number theory and combinatorics. Many classes of numerical semigroups are defined translating ring concepts (see e.g. [2]); for example symmetric and pseudo-symmetric numerical semigroups

\footnotetext{
*e-mail: strazzanti@mail.dm.unipi.it
} 
are the corresponding concepts of Gorenstein and Kunz rings in numerical semigroup theory. Almost symmetric numerical semigroups, that are the object of this paper, were introduced in [3], together with the corresponding notion of almost Gorenstein rings, as generalization of symmetric and pseudosymmetric numerical semigroups; in fact these classes are exactly the almost symmetric numerical semigroups of type 1 and 2, respectively.

In [15] Rosales, García-Sánchez, García-García, and Urbano-Blanco introduced the concept of one half of a numerical semigroup in order to solve proportionally modular diophantine inequalities; $S$ is one half of $T$ if $S=$ $\{s \in \mathbb{N} \mid 2 s \in T\}$. In the last ten years several authors have studied this concept and its generalizations, see for example [5, 7, 8, 16] and the papers quoted below.

Rosales and García-Sánchez proved in [13] that every numerical semigroup is one half of infinitely many symmetric semigroups and Swanson generalized this result in [17]. Moreover Rosales proved in [10] that a numerical semigroup (different from $\mathbb{N}$ ) is one half of a pseudo-symmetric semigroup if and only if is symmetric or pseudo-symmetric.

In this paper we generalize these results to the case of almost symmetric semigroups. According to results of [10], [12], and [13] we consider separately the cases of almost symmetric semigroups with even and odd type.

Starting with a numerical semigroup $S$ with type $t$, in [4] are constructed infinitely many almost symmetric semigroups with type $1,3,5,7, \ldots, 2 t+1$, such that $S$ is their half. This construction is called numerical duplication with respect to a proper ideal of $S$ and arises in commutative algebra, in fact it is the value semigroup of particular algebroid branches (see [1, Theorems 3.4 and 3.6]). In this paper we prove that if $S$ is one half of almost symmetric semigroup $T$ with odd type, then the type of $T$ is included in the values above and all such semigroups can be constructed with the numerical duplication with respect to a relative ideal.

On the other hand if $T$ is almost symmetric with even type, $S$ is almost symmetric and its type is the number of even pseudo-Frobenius numbers of $T$; in particular $t(S) \leq t(T)$. Moreover we prove that a numerical semigroup different from $\mathbb{N}$ is almost symmetric if and only if it is one half of an almost symmetric semigroup with even type or equivalently of a finite number of almost symmetric semigroups with even type. Finally, we characterize these semigroups.

The paper is organized as follows. In the Section 2 we recall some defini- 
tions and results about numerical semigroups and prove some useful lemmas. In Section 3 we introduce the numerical duplication, prove that every numerical semigroups can be realized as numerical duplication with respect to a relative ideal (see Proposition 3.3), and we use this fact in Theorem 3.7 to characterize one half of almost symmetric numerical semigroup $T$ with odd type; moreover, in Theorem 3.1 we give bounds for the type of $T$ (see also the discussion after the theorem). Finally, in the last section we characterize when $T$ is almost symmetric with even type in terms of properties of $\frac{T}{2}$ (see Theorem 4.6) and prove in Corollary 4.12 that a numerical semigroup $S \neq \mathbb{N}$ is almost symmetric if and only if it is one half an almost symmetric numerical semigroup with even type.

\section{Preliminaries}

Let $S$ be a numerical semigroup. The maximum of $\mathbb{N} \backslash S$ is called Frobenius number of $S$ and we denote it by $f(S)$. Clearly if $s \in S \backslash\{0\}$ then $s+f(S) \in S$; more generally we define the set of pseudo-Frobenius numbers $\operatorname{PF}(S)=\{x \in$ $\mathbb{Z} \backslash S \mid x+s \in S$ for any $s \in S \backslash\{0\}\}$. The cardinality of $\operatorname{PF}(S)$ is called the type of $S$; this name is due to ring theory (see e.g. the first section of [2, Chapter II]).

Let $s$ be an integer such that $s \notin S$. If $f(S)-s \in S, s$ is called a gap of first type, otherwise a gap of second type. We denote the set of gaps of second type with $\mathrm{L}(S)$; it is easy to see that $\mathrm{PF}(S) \subseteq \mathrm{L}(S) \cup\{f(S)\}$.

If we have that $s \in S$ if and only if $f(S)-s \notin S$, then we say that $S$ is symmetric; if $f(S)$ is even and this property holds for any $s \in \mathbb{Z}$ but $f(S) / 2$, we call $S$ pseudo-symmetric. Clearly these properties mean that $\mathrm{L}(S)=\emptyset$

and $\mathrm{L}(S)=\left\{\frac{f(S)}{2}\right\}$ respectively. Finally if $\mathrm{L}(S) \subseteq \operatorname{PF}(S)$, we call $S$ almost symmetric. It is well known that $S$ is symmetric if and only if has type 1 , while the pseudo-symmetric numerical semigroups have type 2 (but there are numerical semigroups with type 2 that are not pseudo-symmetric). Almost symmetric semigroups generalize these two classes, in particular symmetric and pseudo-symmetric numerical semigroups are exactly almost symmetric semigroups with type 1 and 2 respectively (see [3, Proposition 7]).

A relative ideal of $S$ is a set $E \subseteq \mathbb{Z}$ such that $E+S \subseteq E$ and $x+E \subseteq S$ for some $x \in S$; moreover if $E \subseteq S$, we say simply that $E$ is a (proper) ideal of $S$. We denote with $f(E)$ the Frobenius number of $E$, i.e. the maximum of $\mathbb{Z} \backslash E$. For example $M(S)=S \backslash\{0\}$ and $K(S)=\{x \in \mathbb{Z} \mid f(S)-x \notin S\}$ are 
relative ideals of $S$ (the first one is a proper ideal) and are called maximal ideal and standard canonical ideal, respectively. More generally we say that $E$ is a canonical ideal of $S$ if $E=K(S)+x$ for some $x \in \mathbb{Z}$. The names of these two ideals come from ring theory and they are very important, for example it is known that $S$ is symmetric if and only if $S=K(S)$ and it is almost symmetric if and only if $M(S)+K(S) \subseteq M(S)$ (see [3, Proposition $4])$. We note the analogy with ring theory, where a Cohen Macaulay local ring $(R, \mathfrak{m})$ is Gorenstein if and only if is isomorphic to its canonical module $K$, and is almost Gorenstein when $\mathfrak{m}+K \subseteq \mathfrak{m}$, where $R \subseteq K \subseteq \bar{R}$.

If $E$ and $F$ are relative ideals of $S$, we define $E-F=\{x \in \mathbb{Z} \mid x+f \in$ $E$ for any $f \in F\}$, that is also a relative ideal. For example we can define $M(S)-M(S)$ in this way and it is easy to check that this is a numerical semigroup satisfying the equality $M(S)-M(S)=S \cup \operatorname{PF}(S)$.

There exist other characterizations of almost symmetric numerical semigroups; in the following sections we will use the next two.

Lemma 2.1. Let $S$ be a numerical semigroup with Frobenius number $f$. Then $S$ is almost symmetric if and only if the following property holds,

$$
s \in S \Longleftrightarrow f-s \notin S \cup \operatorname{PF}(S)
$$

for any $s \in \mathbb{Z} \backslash\{0\}$.

Proof. First of all let $s$ be an element of $S$. One has $f-s \notin S \cup \operatorname{PF}(S)$ or otherwise $f=s+(f-s) \in S$, a contradiction. This is always true.

Now let $s \notin S$. The condition above is equivalent to $f-s \in S \cup \operatorname{PF}(S)$. Clearly, the set of elements such that $f-s \notin S$ for some $s \notin S$ is $\mathrm{L}(S)$, then this condition is equivalent to $\mathrm{L}(S) \subseteq \mathrm{PF}(S)$, that is $S$ is almost symmetric.

The next theorem was proved by Nari in [9], Theorem 2.4.

Theorem 2.2. Let $S$ be a numerical semigroup and $\operatorname{PF}(S)=\left\{f_{1}<\cdots<\right.$ $\left.f_{t-1}<f\right\}$, then the following conditions are equivalent:

(1) $S$ is almost symmetric;

(2) $f_{i}+f_{t-i}=f$ for any $i \in\{1, \ldots, t-1\}$.

In the rest of the paper we distinguish between almost symmetric numerical semigroups with odd and even type. Luckily from [11, Theorem 3] can be deduced a nice distinction between them; here we give a direct proof of this fact. 
Proposition 2.3. Let $T$ be an almost symmetric numerical semigroup, then $T$ has odd type if and only if $f(T)$ is odd.

Proof. Let $\operatorname{PF}(S)=\left\{f_{1}<\cdots<f_{t-1}<f\right\}$. By Theorem 2.2 one has $f_{i}+f_{t-i}=f$, then $t$ is even if and only if $f / 2 \in \operatorname{PF}(S)$. Consequently if $f$ is odd then $t$ is odd. Conversely suppose that $f$ is even; note that $f / 2 \notin S$, otherwise the Frobenius number is in $S$, and that, by Lemma 2.1, $f / 2=f-f / 2 \in S \cup \operatorname{PF}(S)$; hence $f / 2 \in \operatorname{PF}(S)$.

Finally we remember that a numerical semigroup $S$ is one half of $T$ if $S=\{s \in \mathbb{N} \mid 2 s \in T\}$ and in this case we will write $S=\frac{T}{2}$.

\section{One half of almost symmetric numerical semigroups with odd type}

In this section we study those numerical semigroups that are one half of almost symmetric semigroups with odd type.

Theorem 3.1. Let $T$ be an almost symmetric numerical semigroups with odd type $t$. If $S$ is one half of $T$ then $t(S) \geq(t-1) / 2$.

Proof. Let $\mathrm{PF}(T)=\left\{f_{1}<\cdots<f_{t-1}<f\right\}$; by Theorem 2.2 one has $f_{i}+$ $f_{t-i}=f$. In particular, since $f$ is odd, in $\mathrm{PF}(T)$ there are $(t-1) / 2$ even elements and $1+(t-1) / 2$ odd elements.

Consider an even pseudo-Frobenius number $f_{i}=2 e_{i}$; clearly $e_{i} \notin S$ and we claim that $e_{i} \in \operatorname{PF}(S)$. Let $s \in S \backslash\{0\}$, then $2 s \in T$ and therefore $2\left(e_{i}+s\right)=f_{i}+2 s \in T$, because $f_{i} \in \mathrm{PF}(T)$; consequently $e_{i}+s \in S$ for any $s \in S \backslash\{0\}$, that is $e_{i} \in \operatorname{PF}(S)$.

Hence there are at least $(t-1) / 2$ pseudo-Frobenius numbers in $S$.

Remark 3.2. In general, there is not an upper bound for $t(S)$. In fact, in [12] is proved that every numerical semigroup is one half of a symmetric numerical semigroup; then, even if we restrict to the case $T$ symmetric, $S$ may be any numerical semigroup.

Let $S$ be a numerical semigroup, $E$ a proper ideal of $S$ and $b$ an odd element of $S$. In [4] is defined the numerical duplication of $S$ with respect to $E$ as the numerical semigroup

$$
S \bowtie^{b} E=2 \cdot S \cup(2 \cdot E+b)
$$


where $2 \cdot S=\{2 s \mid s \in S\}$ and $2 \cdot E=\{2 e \mid e \in E\}$.

This construction is motivated by a commutative algebra construction (see [1]), but we are interested in it because can be used to construct almost symmetric semigroups. For example Corollary 4.9 of [4] shows that, starting with a numerical semigroup $S$, it is possible to choose proper ideals $E_{0}, E_{1}, \ldots, E_{t(S)}$, such that $S \bowtie^{b} E_{0}, \ldots, S \bowtie^{b} E_{t(S)}$ are almost symmetric of type $1,3,5, \ldots, 2 t(S)+1$, respectively.

Coming back to Theorem 3.1, the inequality of the statement is equivalent to $t \leq 2 t(S)+1$, so the previous remark shows that this estimation is sharp.

Unfortunately there exist almost symmetric numerical semigroups with odd type that cannot be constructed in this way. For example consider $T=\langle 9,10,14,15\rangle=\{0,9,10,14,15,18,19,20,23,24,25,27,28,29,30,32 \rightarrow$ \} , where $\rightarrow$ means that all integers greater than 32 are in $T$; in this case $S=\frac{T}{2}=\{0,5,7,9,10,12,14 \rightarrow\}$. The point is to choose $b \in S$. We must have $2 \cdot E+b=\{9,15,19,23,25,27,29,33,35,37 \ldots\}$, so we have

$$
\begin{array}{ll}
E=\{2,5,7,9,10,11,12,14 \rightarrow\} & \text { if } b=5 \\
E=\{1,4,6,8,9,10,11,13 \rightarrow\} & \text { if } b=7 \\
E=\{0,3,5,7,8,9,10,12 \rightarrow\} & \text { if } b=9 \\
E \text { contains a negative element } & \text { if } b>9
\end{array}
$$
$S$.

in any case $E$ is not contained in $S$ and then $E$ is not a proper ideal of

To solve this problem note that, if $E$ is not a proper ideal, but a relative ideal such that $b+E+E \subseteq S$, then $S \bowtie^{b} E$ is still a numerical semigroup. Under this easy generalization, we can construct all numerical semigroups.

Proposition 3.3. Every numerical semigroup $T$ can be realized as numerical duplication $S \bowtie^{b} E$, where $S=\frac{T}{2}, b$ is an odd element of $S$ and $E$ is a relative ideal of $S$ such that $b+E+E \subseteq S$.

Proof. Let $b$ be an odd element of $S$ and set $E=\frac{T-b}{2}$ (it is possible that $T-b$ contains negative elements and in this case we have negative elements in $\left.\frac{T-b}{2}\right)$. Suppose that $E$ is not a relative ideal of $S$, that is, there exist $s \in S$ and $e \in E$ such that $s+e \notin E$; consequently $2(s+e)+b \notin T$, but $2 s+(2 e+b) \in T+T \subseteq T$, since $s \in S$ and $e \in E$; contradiction. This means that $E$ is a relative ideal of $S$. 
Let $e, e^{\prime}$ be two elements of $E$, then $b+2 e$ and $b+2 e^{\prime}$ is in $T$; therefore $2 b+2 e+2 e^{\prime} \in T$ and it is equivalent to $b+e+e^{\prime} \in S$. Hence $b+E+E \subseteq S$.

Finally, by construction, it is clear that $T=S \bowtie^{b} E$.

Note that in the previous proof we have not determined $b$, so there exist infinitely many ways to obtain the semigroup $T$ as a numerical duplication.

The following corollary is straightforward.

Corollary 3.4. Let $S$ be a numerical semigroup. Then every semigroup $T$ such that $S=\frac{T}{2}$, is equal to $S \bowtie^{b} E$ for some relative ideal $E$ and an odd integer $b \in S$.

Theorem 4.3 of [4] characterizes almost symmetric semigroups realized as numerical duplication with respect to a proper ideal $E$. We will see that in our case only one implication is true.

Let $\widetilde{E}$ denote the relative ideal $E-e$, where $e:=f(E)-f(S)$; clearly $f(\widetilde{E})=f(S)$. Moreover, we set $f:=f(S), M:=M(S)$ and $K:=K(S)$.

First of all we note that, by definition, the Frobenius number of $T=$ $S \bowtie^{b} E$ is the maximum between $2 f(S)$ and $2 f(E)+b$. If $E$ is proper, then $f(E) \geq f(S)$ and so $f(T)=2 f(E)+b$; in general it is possible that $2 f(S)>2 f(E)+b$ : in fact, by Proposition 3.3, $f(T)$ can be even. However, if $T$ is an almost symmetric numerical semigroup with odd type, we have $f(T)=2 f(E)+b$ by Proposition 2.3. Thanks to this, the proof of one implication of [4, Theorem 4.3] works also if $E$ is a relative and not proper ideal of $S$ (obviously provided $E+E+b \subseteq S$ ); hence we will not write the proof of the following proposition.

Proposition 3.5. Let $T$ be an almost symmetric numerical semigroup with odd type. Then there exist a numerical semigroup $S$, a relative ideal $E$ of $S$ and an odd integer $b \in S$ such that $T=S \bowtie^{b} E$. Moreover for every choose of such $S, E, b$ one has $f(T)=2 f(E)+b, K-(M-M) \subseteq \widetilde{E} \subseteq K$, and $K-\widetilde{E}$ is a numerical semigroup.

The converse of the previous result is not true. Consider the numerical semigroup $S=\{0,4,5,6,8 \rightarrow\}$ and the relative ideal $E=\{2,3,4,6 \rightarrow\}$. It is straightforward to check that $K-(M-M)=M=\widetilde{E}, K=S, E+E+5 \subseteq$ $S, K-\widetilde{E}=M-M$ and $2 f(E)+5>2 f(S)$; then $T=S \bowtie^{5} E$ is a numerical semigroup, $K-(M-M) \subseteq \widetilde{E} \subseteq K$ and $K-\widetilde{E}$ is a numerical semigroup. However $T=\{0,8,9,10,11,12,13,16 \rightarrow\}$ is not almost symmetric because $1 \in \mathrm{L}(T) \backslash \mathrm{PF}(T)$. 
If we look at the proof of [4, Theorem 4.3], we see that it works also when $E$ is a relative ideal except for case (iii). From this observation it comes out the idea of the next theorem.

First of all, we recall and generalize some results of [4]. The standard canonical ideal of $S \bowtie^{b} E$ is the set of elements

$$
z=f\left(S \bowtie^{b} E\right)-a \text { with } \begin{cases}\frac{a}{2} \notin S, & a \text { even, } \\ \frac{a-b}{2} \notin E, & a \text { odd. }\end{cases}
$$

The next lemma is proved in [4, Lemma 4.1 and Lemma 4.2]; in the original statement $E$ is a proper ideal, but the proof works also when $E$ is a relative ideal.

Lemma 3.6. Let $E$ be a relative ideal of $S$. Assume that $K-(M-M) \subseteq \widetilde{E}$. Then we have:

(1) for any $x \notin E, f(E)-x \in M-M$;

(2) if moreover $K-\widetilde{E}$ is a numerical semigroup, then, for any $x \notin E$, $f(E)-x \in E-E$.

Now we can construct every almost symmetric numerical semigroup with odd type.

Theorem 3.7. A numerical semigroup $T$ is almost symmetric with odd type if and only if there exist a relative ideal $E$ of $S:=\frac{T}{2}$ and an odd integer $b \in S$ such that:

(1) $T=S \bowtie^{b} E$;

(2) $f(T)=2 f(E)+b$;

(3) $K-(M-M) \subseteq \widetilde{E} \subseteq K$;

(4) $K-\widetilde{E}$ is a numerical semigroup;

(5) $b+e+E+K \subseteq M$.

Proof. As we said above, the proof is only a modification of the proof of [4. Theorem 4.3]. However we include the complete proof for the sake of completeness.

Assume that the five conditions of the statement hold and prove that $T$ is almost symmetric, i.e. $M(T)+K(T) \subseteq M(T)$. There are four cases:

(i) $2 s \in M(T)$ and $2 f(E)+b-a \in K(T)$, where $s \in M, a$ is even and $\frac{a}{2} \notin S$; 
(ii) $2 s \in M(T)$ and $2 f(E)+b-a \in K(T)$, where $s \in M, a$ is odd and $\frac{a-b}{2} \notin E$;

(iii) $2 t+b \in M(T)$ and $2 f(E)+b-a \in K(T)$, where $t \in E, a$ is even and $\frac{a}{2} \notin S$;

(iv) $2 t+b \in M(T)$ and $2 f(E)+b-a \in K(T)$, where $t \in E, a$ is odd and $\frac{a-b}{2} \notin E$.

(i) Since $2 s+2 f(E)+b-a$ is odd, it belongs to $M(T)$ if and only if $s+f(E)-\frac{a}{2} \in E$, i.e. $s+f-\frac{a}{2} \in \widetilde{E}$. Since $\frac{a}{2} \notin S$, i.e. $f-\frac{a}{2} \in K$, we obtain $s+f-\frac{a}{2} \in M+K \subseteq K-(M-M) \subseteq \widetilde{E}$.

(ii) Since $2 s+2 f(E)+b-a$ is even, it belongs to $M(T)$ if and only if $s+f(E)-\frac{a-b}{2} \in M$. Since $\frac{a-b}{2} \notin E$, we can apply Lemma 3.6 to obtain $f(E)-\frac{a-b}{2} \in M-M$, that implies the thesis.

(iii) Since $2 t+b+2 f(E)+b-a$ is even, it belongs to $M(T)$ if and only if $t+b+f(E)-\frac{a}{2} \in M$, i.e. $t+b+e+f-\frac{a}{2} \in M$. But this is true by Condition 5, indeed $t \in E$ and $f-\frac{a}{2} \in K$.

(iv) Since $2 t+b+2 f(E)+b-a$ is odd, it belongs to $M(T)$ if and only if $t+f(E)-\frac{a-b}{2} \in E$. Since $\frac{a-b}{2} \notin E$, the thesis follows immediately by Lemma 3.6 .

This proves that $T$ is almost symmetric; moreover, by Proposition 2.3 its type is odd.

Conversely, we have already seen in Proposition 3.5 that the first four conditions hold. Moreover, it is easy to see that the last one is true: in fact we can use the same argument of case (iii) above.

Remark 3.8. If the conditions of the previous theorem are satisfied, then $E=e+\widetilde{E} \subseteq e+K$; consequently, thanks to the fifth condition, we always have $E+E+b \subseteq S$.

Anyway we notice that there are not problems when $E$ is a canonical ideal, in fact, if we consider only semigroups with odd Frobenius number, the proof of [4, Proposition 3.1] still works. So we have the following theorem:

Theorem 3.9. The numerical semigroup $S \bowtie^{b} E$ is symmetric if and only if $2 f(E)+b>2 f(S)$ and $E$ is a canonical ideal of $S$.

Corollary 3.10. Let $S$ be a numerical semigroup. Then the family of all symmetric numerical semigroups $T$ such that $S=\frac{T}{2}$ is

$$
\mathcal{D}(S)=\left\{S \bowtie^{b} E \mid E+E+b \subseteq S \text { and } E \text { is a canonical ideal of } S\right\}
$$


Proof. By Proposition 3.3, all semigroups can be realized as numerical duplication with respect to a relative ideal $E$ such that $E+E+b \subseteq S$. Hence, recalling Corollary 3.4, we can use the previous theorem.

Finally note that if $E=K+x$, then $E+E+b \subseteq S$ implies $2 x+b>0$, because $0 \in K$. Since $f(E)=f(K)+x=f+x$, we have $2 f<2(f+x)+b=$ $2 f(E)+b$.

Notice that $\mathcal{D}(S)$ is constructed by Rosales and García-Sánchez in [13] in a different way, but it is easy to see that they coincide.

\section{One half of almost symmetric numerical semigroups with even type}

In this section we study when $T$ is almost symmetric with even type or, equivalently, with even Frobenius number.

Lemma 4.1. Let $T$ be a numerical semigroup and $\mathrm{PF}(T)=\left\{f_{1}<\cdots<f_{t}\right\}$. Set $S:=\frac{T}{2}$.

(1) If $f_{i}$ is even, then $\frac{f_{i}}{2} \in \mathrm{PF}(S)$. In particular, the type of $S$ is greater than or equal to the number of even pseudo-Frobenius numbers of $T$.

(2) If $f_{t}$ is even, then $f(S)=\frac{f_{t}}{2}$.

Proof. (1) If $f_{i}$ is even then $\frac{f_{i}}{2} \in \mathbb{Z} \backslash S$, since $f_{i} \notin T$. Let $s$ be a positive element of $S$, then $2 s \in T$ and $f_{i}+2 s \in T$, since $f_{i} \in \operatorname{PF}(T)$; hence $\frac{f_{i}}{2}+s \in S$ and then $\frac{f_{i}}{2} \in \operatorname{PF}(S)$.

(2) See [14, Lemma 6.9].

In [10] is proved that one half of a pseudo-symmetric numerical semigroup is symmetric or pseudo-symmetric; we also know that these classes consist of the almost symmetric semigroup with type 1 or 2 , respectively. In the next theorem this result is generalized for any almost symmetric numerical semigroup with even type.

Theorem 4.2. If $T$ is almost symmetric with even Frobenius number, then $S:=\frac{T}{2}$ is almost symmetric and its type is exactly the number of even pseudoFrobenius numbers of $T$. 
Proof. Let $\mathrm{PF}(S)=\left\{f_{1}<\cdots<f_{t}\right\}$ and $i \in\{1, \ldots, t\}$. Since $f_{i} \notin S, 2 f_{i} \notin T$ and then, thanks to Lemma 2.1 and to Lemma 4.1, $2\left(f_{t}-f_{i}\right)=2 f_{t}-2 f_{i}=$ $f(T)-2 f_{i} \in T \cup \operatorname{PF}(T)$.

If $2\left(f_{t}-f_{i}\right) \in T$, then $s=f_{t}-f_{i} \in S$ and therefore $f_{t}=f_{i}+s$. If $s \neq 0$, then $f_{t} \in S$, since $f_{i} \in \operatorname{PF}(S)$; hence $s=0$, that is $f_{t}=f_{i}$.

Consequently if $i \in\{1, \ldots, t-1\}, 2 f_{t-1}=2\left(f_{t}-f_{i}\right) \in \mathrm{PF}(T)$. In this way we obtain $t-1$ even pseudo-Frobenius number; therefore, since $2 f_{t}(S)$ is not included in this list, there are at least $t$ even pseudo-Frobenius numbers in $T$, and, by the previous lemma, they are exactly $t$.

Finally using Theorem 2.2 it is straightforward to check that $S$ is almost symmetric.

As in the previous section, starting with a numerical semigroup $S$, we can construct all almost symmetric numerical semigroups $T$ with even type, such that $S=\frac{T}{2}$; for this aim we use numerical duplication again.

In [4] this is not possible, because the Frobenius number of the numerical duplication with respect to a proper ideal is always odd.

As in the previous section, $S$ will be a numerical semigroup and we set $f:=f(S), M:=M(S)$ and $K:=K(S)$.

Let us start with some lemmas. The first one was proved by Jäger; for the proof see [6, Hilfssatz 5].

Lemma 4.3. For any relative ideal $E, K-E=\{x \in \mathbb{Z} \mid f-x \notin E\}$.

Lemma 4.4. Suppose that $S$ is almost symmetric and $E$ a relative ideal such that $E+E+b \subseteq S$. Assume that $2 f \geq 2 f(E)+b$. Then the following conditions are equivalent:

(1) $\mathrm{PF}(S) \subseteq E-E$.

(2) $M-M \subseteq E-E$.

(3) $K \subseteq E-E$.

Proof. First we claim that $f \in E-E$. Suppose that there exists $e \in E$ such that $f+e \notin E$. We have $2 e+b>0$, since it is odd and $E+E+b \subseteq S$. Therefore $2 f<2(f+e)+b \leq 2 f(E)+b$. Hence we get a contradiction, since $2 f(E)+b<2 f$.

Now, by definition of almost symmetric semigroups, we have:

$$
M-M=S \cup \operatorname{PF}(S)=S \cup \mathrm{L}(S) \cup\{f\}=K \cup\{f\} .
$$


Moreover, $S \subseteq E-E$ because $E$ is an ideal, then, since $f \in E-E$, we obtain:

$$
\begin{gathered}
\operatorname{PF}(S) \subseteq E-E \Longleftrightarrow S \cup \operatorname{PF}(S) \subseteq E-E \Longleftrightarrow \\
\Longleftrightarrow M-M \subseteq E-E \Longleftrightarrow K \cup\{f\} \subseteq E-E \Longleftrightarrow K \subseteq E-E .
\end{gathered}
$$

Lemma 4.5. Let $S$ be almost symmetric and $E$ a relative ideal. If the equivalent conditions of the previous lemma hold, then $M-E=K-E$.

Proof. Since $M \subseteq K$, one has $M-E \subseteq K-E$. Suppose that the equality does not hold, i.e. there exists $x \in(K-E) \backslash(M-E)$; then there exists $e \in E$ such that $x+e \in K \backslash M$. Since $x+e \in K$, one has $f-x-e \notin S$ and then $f-x-e \in \mathrm{L}(S) \cup\{f\}=\operatorname{PF}(S) \subseteq E-E$ by assumptions. Hence $f-x=(f-x-e)+e \in E$ and, since $x \in K-E$, we obtain $f=(f-x)+x \in K$ that is a contradiction.

Theorem 4.6. Let $S$ be a numerical semigroup, let $b \in S$ be an odd integer, and let $E$ be a relative ideal of $S$ such that $E+E+b \subseteq S$ and $2 f>2 f(E)+b$. Then the numerical duplication $T:=S \bowtie^{b} E$ is almost symmetric (with even type) if and only if the following properties hold:

(i) $S$ is almost symmetric;

(ii) $M-E \subseteq(E-M)+b$;

(iii) $K \subseteq E-E$.

Proof. $T$ is almost symmetric if and only if $M(T)+K(T) \subseteq K(T)$ and, recalling the characterization of $K(T)$, given before Lemma 3.6, this is equivalent to the following four conditions:

(i) $2 m+2 f-a \in M(T)$ for any $m \in M$ and $a$ even such that $\frac{a}{2} \notin S$;

(ii) $2 m+2 f-a \in M(T)$ for any $m \in M$ and $a$ odd such that $\frac{a-b}{2} \notin E$;

(iii) $2 e+b+2 f-a \in M(T)$ for any $e \in E$ and $a$ even such that $\frac{a}{2} \notin S$;

(iv) $2 e+b+2 f-a \in M(T)$ for any $e \in E$ and $a$ odd such that $\frac{a-b}{2} \notin E$.

Discussing every condition, we will see that (i), (ii), (iii) are equivalent to the properties listed in the statement, while condition (iv) is always true, if we assume (i) and (iii). The thesis follows immediately from these facts.

(i) We have $2 m+2 f-a \in M(T)$ if and only if $m+f-\frac{a}{2} \in M$, that 
is $f-\frac{a}{2} \in M-M=S \cup \operatorname{PF}(S)$, for any $\frac{a}{2} \notin S$. Thanks to Lemma 2.1, it is equivalent to say that $S$ is almost symmetric.

(ii) In this case $2 m+2 f-a \in M(T)$ if and only if $\frac{2 m+2 f-a-b}{2} \in E$, that is $m+f-\frac{a-b}{2}-b \in E$. This is equivalent to $f-x \in(E-M)+b$, for any $x \notin E$ and then, applying Lemma 4.3 and Lemma 4.5 we obtain $M-E \subseteq(E-M)+b$.

(iii) The property $2 e+b+2 f-a \in M(T)$ is equivalent to $e+f-\frac{a}{2} \in E$, i.e. $f-\frac{a}{2} \in E-E$. Recalling the definition of $K$, it is equivalent to $K \subseteq E-E$. (iv) Finally, $2 e+b+2 f-a \in M(T)$ if and only if $e+f-\frac{a-b}{2} \in M$, i.e. $f-x \in M-E$ for any $x \notin E$. Using Lemma 4.3 it is equivalent to say $K-E \subseteq M-E$ and, by Lemma 4.5, if we assume (i) and (iii) this fact is always true.

Combining the previous theorem with Proposition 3.3 and Proposition 2.3, we obtain the following corollary.

Corollary 4.7. Let $T$ be a numerical semigroup and $S=\frac{T}{2}$. Then $T$ is almost symmetric semigroup with even type if and only if $S$ is almost symmetric and there exist an odd integer $b \in S$ and a relative ideal $E$ of $S$ such that

(1) $T=S \bowtie^{b} E$;

(2) $M(S)-E \subseteq(E-M(S))+b$;

(3) $K(S) \subseteq E-E$;

(4) $2 f(S)>2 f(E)+b$.

We denote by $m(E)$ the smallest integer of $E$.

Lemma 4.8. Let $S$ be a numerical semigroup, $b \in S$ odd and $E$ a relative ideal of $S$ such that $E+E+b \subseteq S$. Then there exist $b^{\prime}$ and $E^{\prime}$ such that $S \bowtie^{b} E=S \bowtie^{b^{\prime}} E^{\prime}$ and the smallest element of $E^{\prime}$ is zero.

Proof. Set $E^{\prime}:=E-m(E)$ and $b^{\prime}:=b+2 m(E)$. Clearly $m\left(E^{\prime}\right)=0$ and $E^{\prime}+E^{\prime}+b^{\prime}=E-m(E)+E-m(E)+b+2 m(E)=E+E+b \subseteq S$; moreover, we have $b^{\prime} \in E+E+b \subseteq S$ and, if $e \in E$, then $2 e+b=2(e-m(E))+b+2 m(E) \in$ $2 E^{\prime}+b^{\prime}$ and vice versa; hence we have the thesis.

Remark 4.9. Let $S$ be an almost symmetric numerical semigroup; we want to know which almost symmetric semigroups $T$ with even type satisfy $S=\frac{T}{2}$. 
Assume that the smallest element of $E$ is zero. According to Corollary 4.7, one has $2 f(S)>2 f(E)+b$, then $b<2 f(S)-2 f(E) \leq 2 f(S)+2$. Hence we have a finite number of possibilities for $b$; moreover, if we fixed $b$, we have $-1 \leq f(E)<f(S)-\frac{b}{2}$ and then there are finitely many choices for $E$.

This fact is obvious, since $f(T)=2 f(S)$ and there is a finite number of semigroups with fixed Frobenius number; however this remark is useful for the next example.

Example 4.10. Consider the pseudo-symmetric semigroup $S=\{0,3,5 \rightarrow\}$; we want to construct all almost symmetric semigroups $T$ with even type such that $S=\frac{T}{2}$.

As in the previous remark we have $b<10$ and, for a fixed $b,-1 \leq f(E)<$ $4-\frac{b}{2}$. In view of Lemma 4.8 we are looking for only ideals containing zero and then $f(E)$ is different from zero. We have four possibilities:

$$
\begin{array}{lll}
b=3 & \Longrightarrow & f(E)=-1,1,2 . \\
b=5 & \Longrightarrow & f(E)=-1,1 . \\
b=7 & \Longrightarrow & f(E)=-1 . \\
b=9 & \Longrightarrow & f(E)=-1 .
\end{array}
$$

The unique ideals with Frobenius number -1 and 1 are, respectively, $E_{1}=\mathbb{N}$ and $E_{2}=\{0,2 \rightarrow\}$, while there are two ideals with Frobenius number $2, E_{3}=\{0,3 \rightarrow\}$ and $E_{4}=\{0,1,3 \rightarrow\}$. Note that, if $b=3, E_{1}$ and $E_{4}$ are not acceptable, because, in this case, $E+E+b \nsubseteq S$. It is straightforward to check that $E_{i}-E_{i}=E_{i}$ for $i=1,2,3$ and then $K=\{0,2,3,5 \rightarrow\}$ is contained in $E_{i}-E_{i}$ for $i=1,2$ but not for $i=3$. Finally we have

$$
\begin{array}{ll}
M-E_{1}=\{5 \rightarrow\}, & E_{1}-M=\{-3 \rightarrow\}, \\
M-E_{2}=\{3,5 \rightarrow\}, & E_{2}-M=\{-3,-1 \rightarrow\}
\end{array}
$$

and then we obtain:

$$
\begin{array}{ll}
b=3 & M-E_{2} \subseteq\left(E_{2}-M\right)+b, \\
b=5 & M-E_{1} \subseteq\left(E_{1}-M\right)+b, \\
& M-E_{2} \nsubseteq\left(E_{2}-M\right)+b, \\
b=7 & M-E_{1} \subseteq\left(E_{1}-M\right)+b, \\
b=9 & M-E_{1} \nsubseteq\left(E_{1}-M\right)+b .
\end{array}
$$

Hence we have three possibilities and they give the numerical semigroups

$$
\begin{aligned}
& S \bowtie^{3} E_{2}=\{0,3,6,7,9 \rightarrow\}, \\
& S \bowtie^{5} E_{1}=\{0,5,6,7,9 \rightarrow\}, \\
& S \bowtie^{7} E_{1}=\{0,6,7,9 \rightarrow\} .
\end{aligned}
$$


Note that the first two are pseudo-symmetric, while the last one is almost symmetric with type four.

Lemma 4.11. For any almost symmetric numerical semigroup $S$ different from $\mathbb{N}$, there exists at least one relative ideal $E$ and one odd integer $b \in S$ such that $S \bowtie^{b} E$ is almost symmetric with even type.

Proof. We set $E:=\mathbb{N}$ and $b:=f+1$ if it is odd, or otherwise $b:=f+2$.

First of all note that if $e, e^{\prime} \in E$, one has $e+e^{\prime}+b>f$, then $E+E+b \subseteq S$; moreover $2 f(E)+b=-2+b \leq-2+f+2<2 f$. Then, by Theorem 4.6, we have to prove that $K \subseteq E-E$ and $M-E \subseteq(E-M)+b=(E+b)-M$. It is straightforward to check that $E-E=\mathbb{N}$ and $M-E=\{f+1 \rightarrow\}$, then clearly $K \subseteq E-E$ and if $m \in M$ and $x \in M-E$ one has $m+x \geq f+2$. Hence $m+x \in\{f+2, \rightarrow\} \subseteq\{b \rightarrow\}=E+b$ and consequently $M-E \subseteq((E+b)-M)$ as required.

We have already seen that one half of an almost symmetric numerical semigroup with even type is almost symmetric and the previous lemma proves the converse: every almost symmetric numerical semigroup is one half of some almost symmetric semigroup with even type. Then we can state the last corollary:

Corollary 4.12. A numerical semigroup different from $\mathbb{N}$ is almost symmetric if and only if it is one half of an almost symmetric numerical semigroup with even type.

Notice that, if $\mathbb{N}$ is one half of a semigroup $T$, then $T$ contains all even positive integer. Hence $f(T)$ is odd and it is easy to see that $T$ is symmetric.

Acknowledgments. The author would like to thank Marco D'Anna for his help and support during the drafting of the paper and Pedro García-Sánchez for his useful suggestions.

\section{References}

[1] V. Barucci, M. D'Anna, F. Strazzanti, A family of quotients of the Rees algebra, Communications in Algebra, DOI:10.1080/00927872.2014.897549.

[2] V. Barucci, D. E. Dobbs, M. Fontana, Maximality properties in numerical semigroups and applications to one-dimensional analytically irreducible local domain, Mem. Amer. Math. Soc. vol. 125, no. 598 (1997). 
[3] V. Barucci, R. Fröberg, One-dimensional almost Gorenstein rings, J. of Algebra 188 (1997), 418-442.

[4] M. D'Anna, F. Strazzanti, Numerical duplication of a numerical semigroup, Semigroup Forum 87 (2013), no. 1, 149-160.

[5] D. E. Dobbs, H. J. Smith, Numerical semigroups whose fractions are of maximal embedding dimension, Semigroup Forum 82 (2011), no.3, 412-422.

[6] J. Jäger, Längenberechnung und kanonische ideale in eindimensionalen ringen, Arch. Math. 29 (1977), 504-512.

[7] V. Micale, A. Olteanu, On the Betti numbers of some semigroup rings, Le Matematiche 67 (2012), no. 1, 145-159.

[8] A. Moscariello, Generators of a fraction of a numerical semigroup, arXiv:1402.4905 1 (2014).

[9] H. Nari, Symmetries on almost symmetric numerical semigroups, Semigroup Forum 86 (2013), no. 1, 140-154.

[10] J. C. Rosales, One half of a pseudo-symmetric numerical semigroup, Bull. Lond. Math. Soc. 40 (2008), no. 2, 347-352.

[11] J. C. Rosales, P. A. García-Sánchez, Constructing Almost Symmetric Numerical Semigroups from Irreducible Numerical Semigroups, Communications in Algebra 42 (2014), no. 3, 1362-1367.

[12] J. C. Rosales, P. A. García-Sánchez, Every numerical semigroup is one half of a symmetric numerical semigroups, Proc. of the Amer. Math. Soc. 136 (2008), no. 2, 475-477.

[13] J. C. Rosales, P. A. García-Sánchez, Every numerical semigroup is one half of infinitely many symmetric numerical semigroups, Communications in Algebra 36 (2008), 2910-2916.

[14] J. C. Rosales, P. A. García-Sánchez, Numerical Semigroups, Springer Developments in Mathematics, Vol 20, 2009.

[15] J. C. Rosales, P. A. García-Sánchez, J. I. García-García, J. M. Urbano-Blanco, Proportionally modular Diophantine inequalities, J. Number Theory 103 (2003), 281294.

[16] H. J. Smith, Numerical semigroups that are fractions of numerical semigroups of maximal embedding dimension, JP J. Algebra Number Theory Appl. 17 (2010), no.1, 69-96. 
[17] I. Swanson, Every numerical semigroup is one over d of infinitely many symmetric numerical semigroups, Commutative algebra and its applications, Walter de Gruyter, Berlin, (2009), 383-386. 